\title{
Review of Basic Concepts Involved in Magnetic Resonance Imaging
}

\author{
Harneet Arora* \\ Department of Physical Therapy, University of Florida, USA
}

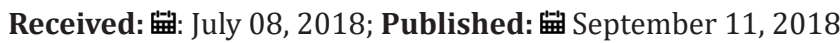

*Corresponding author: Harneet Arora, Department of Physical Therapy, University of Florida, USA

\begin{abstract}
Magnetic resonance imaging (MRI) is a powerful tool to get spatial information about muscles of different regions inside the body. It has the advantage of being non-invasive and being without any radiations. It is objective and quantitative tool. This review covers the basics of MRI and informs about how the image is obtained using MRI.
\end{abstract}

Abbreviations: MRI: Magnetic Resonance Imaging; NMR: Nuclear Magnetic Resonance; MR: Magnetic Resonance; RF: Radiofrequency; MDM: Magnetic Dipole Moment; FID: Free Induction Decay; PD: Proton Density

\section{Introduction}

Magnetic Resonance Imaging (MRI) is a non-invasive imaging technique which forms images of the inside of the body [1,2]. MRI works on the principle of nuclear magnetic resonance (NMR), a phenomenon where nuclei of atoms get excited in the magnetic field by electromagnetic waves and emit signals [3,4]. Magnetic Resonance (MR) was independently developed by Felix Bloch and Edward Purcell in 1946. Bloch and Purcell shared the 1952 Nobel Prize in physics for this discovery [1,4]. Paul Lauterbur generated the first MRI on small test tube samples in 1973 [1]. Lauterbur and Mansfield formed different ways to generate images from magnetic spins [4]. They both were awarded the Nobel Prize in Medicine in 2003 for their discoveries about MRI [1]. Since then, MRI has been an invaluable tool for diagnosis of a number of pathologies in medicine [2,5].

\section{Basics of MRI}

Electromagnetic waves travel at the speed of light $(3 * 108 \mathrm{~m} /$ sec). They form electric and magnetic field components, which are perpendicular to each other. X-Ray, visible light, microwaves, and radio waves are all forms of electromagnetic waves. In MRI, the 3 - $100 \mathrm{MHz}$ range of radiofrequency (RF) pulse, an electromagnetic pulse, is used for signal generation. Bloch hypothesized that when any charged particle spins, it generates an electromagnetic field [3] (Figure 1). An atom may have an even or an odd number of protons. When there is an even number of protons, the paired protons cancel each other's magnetic field resulting in a net zero magnetic field. With an odd number of protons, there is always an existence of one unpaired proton, which produces a net magnetic field or magnetic dipole moment (MDM) to the nucleus. While MRI could use any nuclei with an odd number of protons, hydrogen has been selected for imaging. The reason being that hydrogen is found in abundance in the human body.

Hydrogen is found in water, which is approximately $60 \%$ of the body weight, and in fats [3]. Protons of nuclei spin on their own axes and have their own small magnetic fields or MDMs. When there is no external magnetic field (B0), the axes of these MDMs are randomly arranged. Thus, they cancel each other out resulting in a net zero magnetic field. In the presence of B0, over time, the protons' spins will line themselves up in the direction of B0 and produce a net magnetic field. Protons start to wobble or precess in the presence of B0. Thus, they spin on their own axes as well as precess about the axis of $\mathrm{B} 0$. The rate of precession of protons around B0 is based on the Larmor equation $-\omega=\gamma$ B0

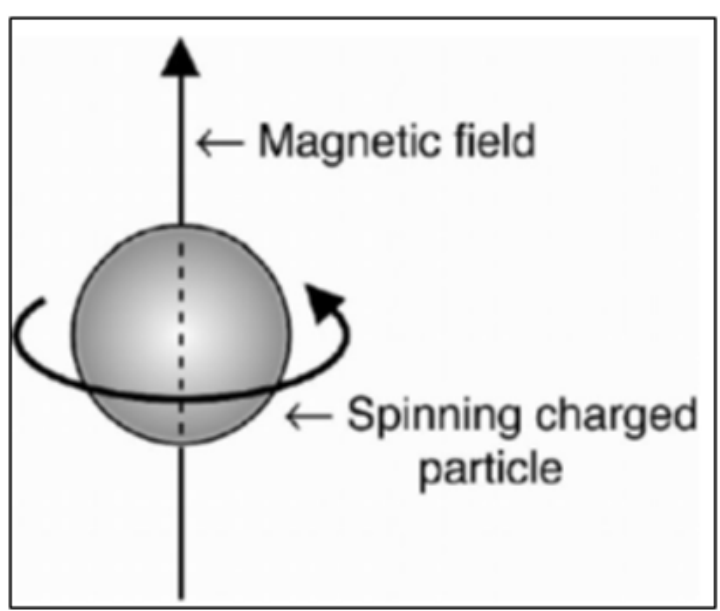

Figure 1: A: Source: Animalia World. B: It's a relay station for everything sensory that comes from the brain. 
Where $\omega=$ angular precessional frequency of a proton (expressed in $\mathrm{MHz}$ ),

\section{$\gamma=$ gyromagnetic ratio (expressed in MHz/Tesla),}

$\mathrm{B} 0=$ strength of the external magnetic field (expressed in Tesla) [3].

When the object to be imaged is placed in B0, the proton spins align longitudinally along the direction of $\mathrm{B} 0$ about which they precess. The magnetization along the direction of $\mathrm{B} 0$ is called longitudinal magnetization, Mz [1,3]. When an RF pulse is applied, this longitudinal magnetization, Mz flips into a transverse plane, creating a transverse magnetization, Mxy (Figure 2) [2,4]. This transverse magnetization will precess around B0 producing an alternating current in a receiver coil and thus, generating a measureable signal [2]. When the RF pulse is turned off, the protons start going back to their lower energy states, realigning in the direction of B0. This causes a decay of magnetization over time, resulting in a decreased signal in the Mxy plane. This decreased amplitude of the signal over a period of time is called free induction decay (FID) (Figure 3) [2]. Two independent relaxation processes occur at the same time, namely, longitudinal relaxation and transverse relaxation [2].

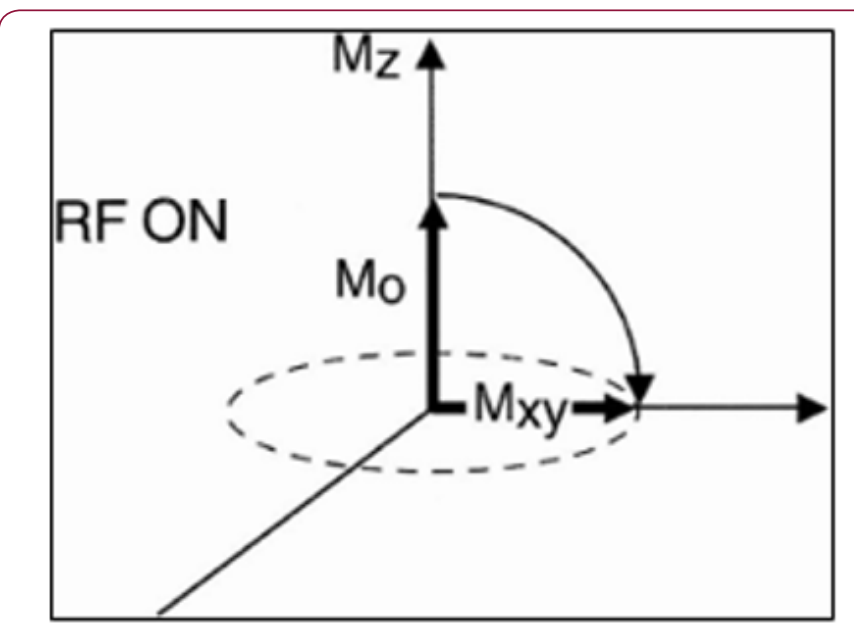

Figure 2: 90degree RF pulse flips longitudinal magnetization into transverse magnetization [3].

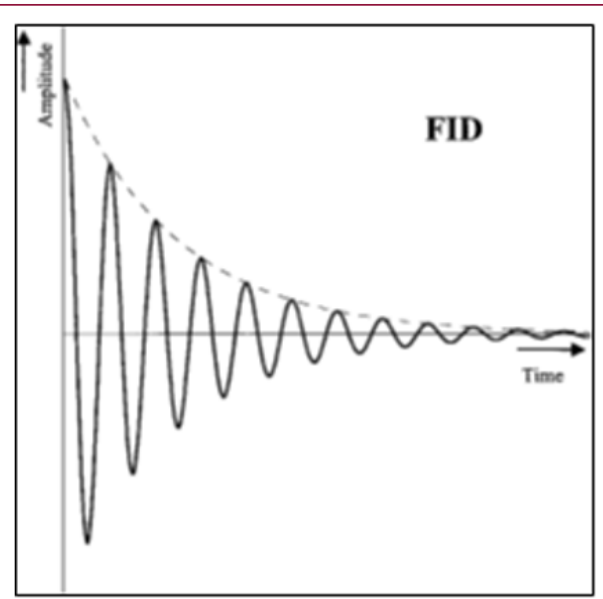

Figure 3: A decay of signal over time is called free induction decay [2].

\section{Relaxation Times}

When the protons' spins go back to their lowest energy state or equilibrium state, it is called relaxation. Relaxation occurs when the RF pulse is turned off and protons realign along the direction of B0, thereby releasing all the extra energy [3]. Relaxation time is the time required by the protons to go back to their lowest energy state [2].

Longitudinal Relaxation Time: Longitudinal relaxation time, $\mathrm{T} 1$, is the time taken by the protons' spins to realign themselves along the direction of the longitudinal (Z) axis [2,3,6]. T1 is also called spin-lattice relaxation time because it also denotes the time required for the excited protons' spins to give back the energy to the surrounding lattice in order to reach an equilibrium state $[3,6]$. Transverse relaxation time: Transverse relaxation time, $\mathrm{T} 2$, is the time taken for decaying of transverse magnetization in the xy plane.

T2 decay occurs 5 to 10 times faster than T1 recovery (Figure 4) [3]. When the RF pulse is turned off, protons' spins will start to dephase because of spin-spin interactions and external magnetic field inhomogeneities [2,3]. T2 is also called spin-spin relaxation time because it is the time taken by transverse magnetization to decay which is caused due to spin-spin interactions. When the effect of external magnetic field inhomogeneities is added to T2 relaxation time, it is called $\mathrm{T} 2 *$ relaxation time [7].

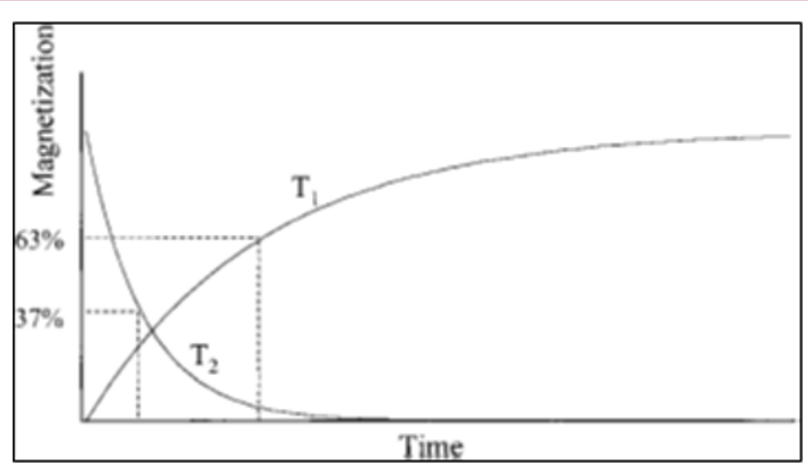

Figure 4: The longitudinal magnetization recovery and transverse magnetization decay occur independently of each other. T1 is the time to regain $63 \%$ of original net magnetization. T2 is the time of signal decay to $37 \%$ of its original signal [2].

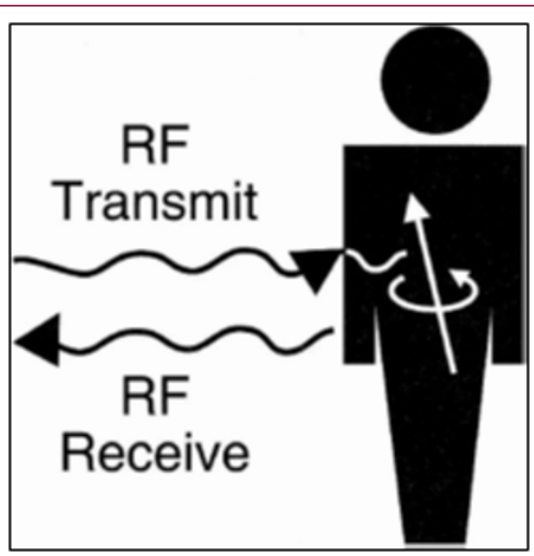

Figure 5: RF pulse is given to the subject and then signal is obtained from magnetized spins in the body [1]. 
Image Formation with MRI: In the presence of B0, unpaired protons' spins line up in the direction of the magnetic field. After the protons' spins line up, an RF pulse is transmitted to the tissues of the body, causing some spins to align in the direction of a new magnetic field. After the RF pulse, magnetized spins in the body produce an MR signal while they return to their original alignment (Figure 5). But the signal obtained contains information from the entire imaged part of the body without any spatial information, so gradients are used to get spatial information. Gradients produce a linear magnetic field non-uniformity along all three axes which helps in obtaining spatial information [3].

There are three different types of gradients used [2-4]:

a) Slice-Selection Gradient - A slice-selection gradient is used to select a particular slice in the body [3]. For imaging a specific slice, an RF pulse of a specific frequency is chosen to only excite the selected slice with the slice-selection gradient in the z- axis [4].

b) Frequency-Encoding Gradient - Once the slice is selected, a frequency-encoding gradient is used along the $\mathrm{x}$-axis after the $\mathrm{RF}$ pulse is applied. It affects the precession frequency of the protons, generating signals of different frequencies, which vary from each other in a predictable manner [4].

c) Phase-Encoding Gradient - A phase-encoding gradient is used for inducing phase differences along the y-axis. It is turned on and off for a short period of time, after the RF pulse is applied and before the application of a frequency-encoding gradient $[4,6]$. When this gradient is turned on, some protons start precessing faster than others, depending on their location in relation to the gradient. When the gradient is switched off, the protons become out of phase from one another. Thus, protons are in different phases along the y-axis [4].

\section{Parameters that Define Contrast in an Image}

Image Contrast: One of the most significant benefits of MRI, particularly when compared to other imaging techniques is its ability to differentiate between various tissues of the body [4,7]. Different signal intensities of different tissues produce an image contrast [8]. Intrinsic parameters of image contrast: T1 and T2 are fixed intrinsic properties of the tissue [3], which have already been described above. Contrast is generated between tissues because of differences in T1 and T2 values [7]. Another parameter which can define an image contrast is the proton density of the tissue [8].

Proton Density: Proton Density (PD) refers to the concentration of hydrogen protons in a tissue. It exists within the tissue and is thus independent of the existence of an external magnetic field. PD usually denotes the number of mobile hydrogen protons present in a tissue and is related to the signal intensity [9].

Extrinsic Parameters of Image Contrast: Image contrast can also be obtained by changing the operator-controlled parameters of MRI: repetition time and echo time.

Repetition Time: Repetition time (TR) is the time interval between two consecutive 90-degree RF pulses.
Echo Time: Echo time (TE) is the time interval between sending the RF pulse and measuring the signal [3]. By changing TR and TE, various "weighted" images can be formed. T1 weighted image: If TR and TE are both short, a T1 weighted image is obtained $[3,10]$. With a short TR, tissues can be distinguished from each other by their different T1s [3] (Figure 6). If TR is long, it would have caused a full recovery of longitudinal magnetization of tissues, resulting in no difference in their T1s. So, a short TR helps in increasing the T1 effect. A short TE results in reduction of the T2 effect $[2,3,6,11,12]$. A T1 weighted image provides good anatomical details of different tissues [6]. Fat has more signal intensity than water as recovery of fat is quicker than that of water [10]. Signal intensity is low for most lesions [10].

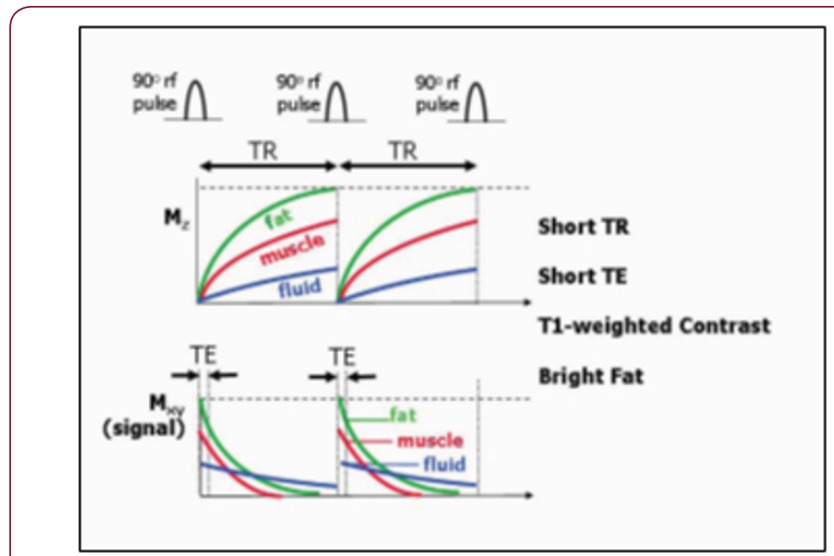

Figure 6: T1 weighted spin echo: T1 and T2 curves for T1 weighted spin echo for three different tissues. T1 weighted images are characterized by a short TR and a short TE. Bright fat signal is seen on these images [7].

T2 Weighted Image: If TR and TE are both long, a T2 weighted image is obtained $[3,9]$. T1 effect is reduced when TR is long $[2,3,12]$. A TR of 2000-3000 msec can be used for decreasing the T1 effect (Hashemi, 2004). A long TE results in more dephasing of protons, which further increases the T2 contrast between tissues (Figure 7) [2,3,6]. Fat has less signal intensity than water as decay of fat is more rapid than that of water [9]. Signal intensity is high for most lesions [10].

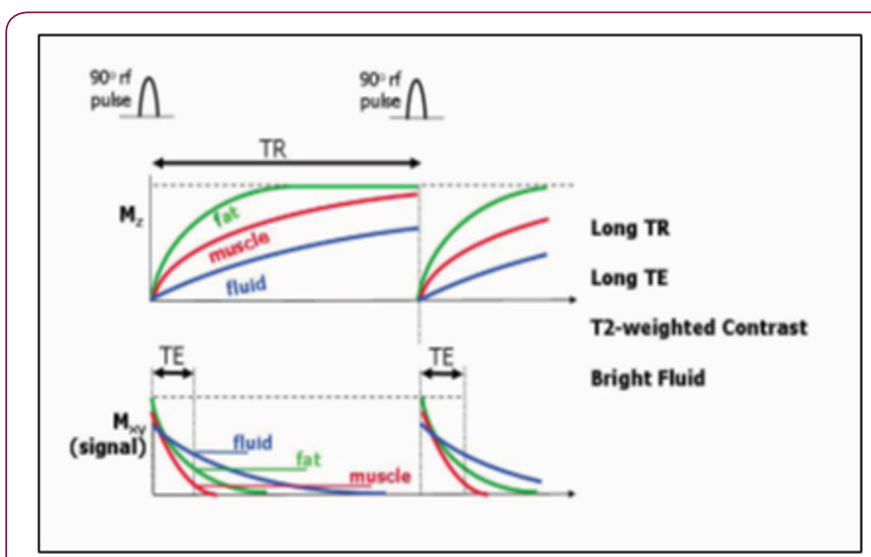

Figure 7: T2 weighted spin echo: $\mathrm{T} 1$ and $\mathrm{T} 2$ curves for $\mathrm{T} 2$ weighted spin echo for three different tissues. T2 weighted images are characterized by a long TR and a long TE. Bright fluid signal is seen on these images [7]. 
Proton Density Weighted Image: By using a long TR and a short TE, a proton density weighted image is produced. A long TR minimizes the T1 effect and a short TE decreases the T2 effect. So, the resultant contrast between the tissues would be based on the proton densities of the tissues $[2,3,12]$.

\section{Conclusion}

Magnetic resonance imaging is an invaluable tool for getting the information about the inside of the body, in a safe way. It has been used for diagnostic and research purposes for a long time. The above review explains about the basic concepts so to have a better understanding about how MRI works.

\section{References}

1. Hornak JP (2010) The Basics of MRI Retrieved from.

2. Van Geuns RJ, Wielopolski PA, De Bruin HG, Rensing BJ (1999) Basic principles of magnetic resonance imaging. Prog Cardiovasc Dias 42(2): 149-156.

3. Hashemi RH, Bradley WG, Lisanti CJ (2004) MRI: The Basics (2 $\left.2^{\text {nd }} e d n.\right)$. Lippincott Williams \& Wilkins.

\section{ISSN: 2574-1241}

DOI: $10.26717 / B J S T R .2018 .08 .001726$

Harneet Arora. Biomed J Sci \& Tech Res

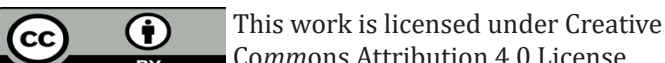

Submission Link: https://biomedres.us/submit-manuscript.php
4. Sands MJ, Levitin A (2004) Basics of magnetic resonance imaging. Semin Vasc Surg 17(2): 66-82.

5. Boesch C (1999) Molecular aspects of magnetic resonance imaging and spectroscopy. Mol Aspects Med 20(4-5): 185-318.

6. McMahon KL, Cowin G, Galloway G (2011) Magnetic resonance imaging: the underlying principles. J Orthop Sports Phys Ther 41(11): 806-819.

7. Ridgway JP (2010) Cardiovascular magnetic resonance physics for clinicians: part I. J Cardiovasc Magn Reson 12: 71.

8. Wehrli FW, Kanal E (1987) Orbital imaging: factors determining magnetic resonance imaging appearance. Radiol Clin North Am 25(3): 419-427.

9. Shah B, Anderson SW, Scalera J, Jara H, Soto JA, et al. (2011) Quantitative MR imaging: physical principles and sequence design in abdominal imaging. Radiographics 31(3): 867-880.

10. Anupindi S, Jaramillo D (2002) Pediatric magnetic resonance imaging techniques. Magn Reson Imaging Clin N Am 10(2): 189-207.

11. Buxton RB, Edelman RR, Rosen BR, Wismer GL, Brady TJ, et al. (1987) Contrast in rapid MR imaging: T1- and T2-weighted imaging. J Comput Assist Tomogr 11(1): 7-16.

12. Makow LS (1989) Magnetic resonance imaging: a brief review of image contrast. Radiol Clin North Am 27(2): 195-218.

$\begin{array}{ll}\text { BIOMEDICAL } & \text { Assets of Publishing with us } \\ \text { RESEARCHES } & \text { - Global archiving of articles } \\ \text { - Immediate, unrestricted online access }\end{array}$

